FACTA UNIVERSITATIS

Series: Mechanical Engineering Vol. 14, N 3 3, 2016, pp. 241 - 249

DOI: $10.22190 /$ FUME1603241L

Original scientific paper

\title{
INDENTATION OF FLAT-ENDED AND TAPERED INDENTERS WITH POLYGONAL CROSS-SECTIONS
}

\author{
UDC 539.3
}

\author{
Qiang Li, Valentin L. Popov \\ Department of System Dynamics and the Physics of Friction, TU Berlin, Germany
}

\begin{abstract}
Using the Boundary Element Method, we numerically study the indentation of prismatic and tapered indenters with polygonal cross-sections. The contact stiffness of punches with flat bases in the form of a triangle and a square as well as a number of higher-order polygons is determined. In particular, the classical results of King (1987) for indenters with triangle and square base shapes are revised and more precise numerical results are provided. For tapered indenters, the equivalent transformed profile used in the Method of Dimensionality Reduction (MDR) is determined. It is shown that the MDR-transformed profile of polygon-based indenters with power function side is given by the power function with the same power; it differs from the $3 D$ profile only by a constant coefficient. These coefficients are listed in the paper for various types of indenters, in particular for pyramidal and paraboloid ones. The determined MDR-transformed profiles can be used for study of other contact problems such as tangential contact, normal contact with elastomers, and, in an approximate way, to adhesive contacts.
\end{abstract}

Key Words: Indentation, Contact Stiffness, Polygonal Indenter, Boundary Element Method, MDR Transformed Profile

\section{INTRODUCTION}

Indentation test is a very common way of probing mechanical properties of materials such as hardness, contact stiffness, elastic modulus and strain-stress relation [1-3]. There is a variety of indenter geometries used in macro- and microindentation; the most popular are spherical and pyramidal indenters (e.g. for the Vickers hardness test and Brinell hardness test) [4]. The contact stiffness of indenters with regular geometries is also important for the foundation design [5]. The analytical solution for contact between a rigid cylindrical flat punch and an elastic half space was given by Galin in 1953 (English translation see [6]). His results were later published by Sneddon and, in this way, made public to the western world [7]. Based on this

Received September 10, 2016 / Accepted November 04, 2016

Corresponding author: Qiang Li

Institute of Mechanics, Berlin Institute of Technology, Strasse des 17. Juni 135, 10623 Berlin, Germany

E-mail: qiang.li@tu-berlin.de 
solution, Oliver and Pharr proposed an analysis method to determine the hardness and elastic modulus from the load-displacement curves of indentation test [8]. General relations among contact stiffness, contact area, and elastic modulus during indentation have been analytically derived only for axisymmetric indenters. For a non-axisymmetrical geometry, a correction coefficient is needed [9], which can be still found only numerically.

In this paper we numerically investigate the indentation of rigid bodies with various geometries: the flat-ended punches in Section 2 and tapered indenters in Section 3. In both cases we consider different polygonal bases including triangle and square. Note that the assumption of a rigid indenter is no restriction as the normal frictionless contact of two elastic bodies with elastic moduli $E_{1}$ and $E_{2}$ and Poisson numbers $v_{l}$ and $v_{2}$ can always be reduced to the contact of a rigid indenter and an elastic medium with an effective elastic modulus $E^{*}$ determined as [10]

$$
\frac{1}{E^{*}}=\frac{1-v_{1}^{2}}{E_{1}}+\frac{1-v_{2}^{2}}{E_{2}} .
$$

In the present paper, the indentation test is numerically simulated by the high resolution Boundary Element Method (BEM), which has recently been generalized to arbitrary contact problems including tangential contact and adhesive contact $[11,12]$.

\section{INDENTATION OF PRISMATIC INDENTERS WITH POLYGONAL BASE}

The normal contact stiffness between a rigid flat cylinder and an elastic half space is given by $k=2 a E^{*}$ [7], where $a$ is the radius of the cylinder, and $E^{*}$ is the effective elastic modulus, Eq. (1). In the case of a prismatic indenter with an arbitrary base form, the normal contact stiffness is given by [5]:

$$
k=\beta \cdot 2 E^{*} \sqrt{\frac{A}{\pi}},
$$

where $A$ is the contact area of the base. Obviously the value of $\beta$ is equal to 1 for the flat-ended cylinder. It was proven that Eq. (2) is also valid for indenters which have a cross section other than a circle [5]: $\beta=1.034$ for triangle and $\beta=1.012$ for square. These results were numerically obtained by King in 1987 . Due to the limitation of computer technology at that time, King used only 200 elements for simulating a triangle indenter, and the

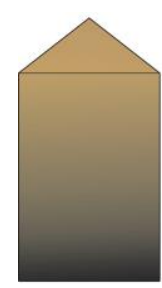

(a)

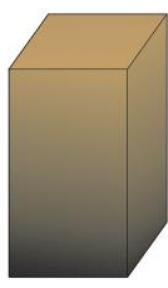

(b)

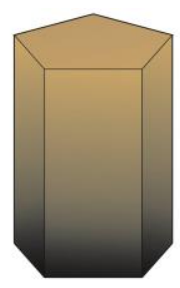

(c)

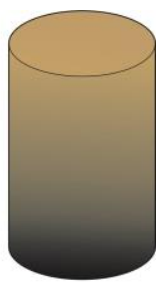

(d)

Fig. 1 Prismatic indenters with polygonal bases: $m=3$ (triangle), $m=4$ (square), $m=5$ (pentagon) and $m=\infty$ (circle) 
triangular area looked quite 'rugged'. Note that the stiffness of a flat punch and correspondingly factor $\beta$ are related to the so-called harmonic capacity of the base form of the punch. This analogy was discussed by Argatov (2010) [13].

Below we repeat the calculations of King using the current high-resolution BEM and provide corrected values.

Using the boundary element method we have numerically carried out the indentation test for different shapes of cross section of indenters: from triangle $(m=3)$, square $(m=4)$, pentagon $(m=5)$ to circle $(m=\infty)$ as shown in Fig. 1. In the simulation, the whole area was divided into $1024 \times 1024$ elements where at least 200000 elements were in the contact area. It is at least 1000 times more than in the King's simulations; therefore, a much more precise result could be obtained. The values of coefficient $\beta$ for different $m$ are presented in Fig.2 and Table1. For the two most popular indenter shapes, the values are:

$$
\begin{aligned}
& \beta=1.061, \text { for triangle, } \\
& \beta=1.021, \text { for square, }
\end{aligned}
$$

which is larger than the values reported by King [5]. It can be seen that with the same area of cross section, the stiffness of triangular indenter is for $6 \%$ larger than that of a flat cylinder.

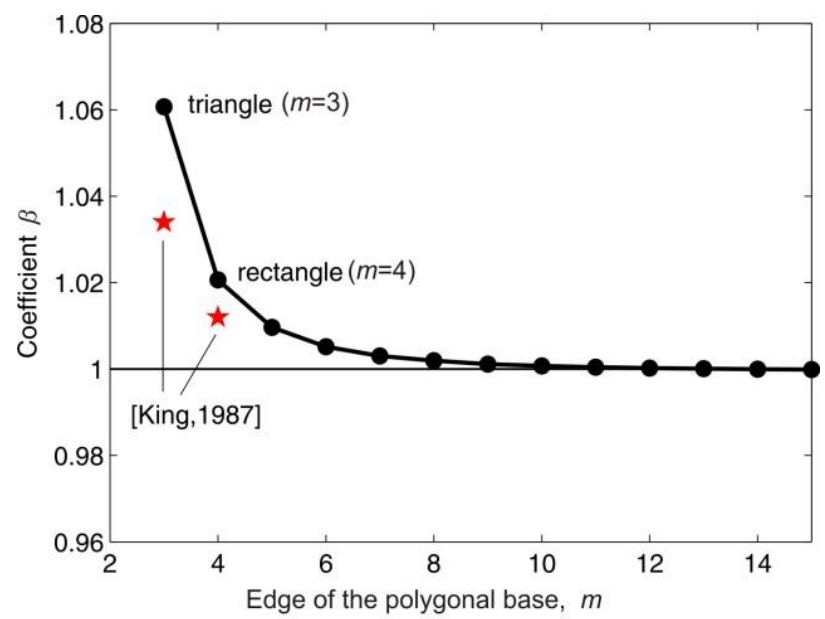

Fig. 2 Factor $\beta$ for different polygonal indenters. The two stars indicate the results obtained numerically by King in 1987 [5]

Table 1 Values of constant $\beta$

\begin{tabular}{cccccccc}
\hline $\begin{array}{c}m \\
\text { polygon }\end{array}$ & $\begin{array}{c}3 \\
\text { (triangle) }\end{array}$ & $\begin{array}{c}4 \\
\text { (square) }\end{array}$ & 5 & 6 & 7 & 8 & $\begin{array}{c}\infty \\
\text { (cylinder) }\end{array}$ \\
\hline$\beta$ & 1.061 & 1.021 & 1.010 & 1.005 & 1.003 & 1.002 & 1.000 \\
\hline
\end{tabular}




\section{INDENTATION OF TAPERED INDENTERS WITH POLYGONAL BASE AND POWER FUNCTION SIDE SURFACE}

Now we consider the tapered indenters which have a regular polygonal base, as shown in Fig. 3. We begin with the most common type - a pyramid, and then extend it to indenters whose side profile is an arbitrary power function.

\subsection{Pyramidal indenters}

For the contact between a rigid cone with profile $f(r)=\tan \theta \cdot r$ and an elastic half space with effective elastic module $E^{*}$, the dependence of normal force on indentation depth was analytically found by Galin [6] (see also Sneddon [7]):

$$
F_{N}=\frac{2}{\pi} \frac{E^{*}}{\tan \theta} d^{2},
$$

where $d$ is indentation depth and $\theta$ is defined in Fig. 3(c). This solution can be easily reproduced using the method of dimensionality reduction (MDR). In the framework of the MDR [14], any contact problem of an axis-symmetrical profile $f(r)$ with an elastic half-space can be mapped onto a contact of a modified (MDR-transformed) profile $g(x)$ :

$$
g(x)=|x| \int_{0}^{|x|} \frac{f^{\prime}(r)}{\sqrt{x^{2}-r^{2}}} \mathrm{~d} r,
$$

with properly defined elastic foundation. For a conical profile, $f(r)=\tan \theta \cdot r$, the substitution in Eq. (5) and integration provides the MDR-transformed profile:

$$
g(x)=(\pi / 2) \cdot|x| \cdot \tan \theta .
$$

A short calculation (see. e.g. [14]) leads to Eq. (4).

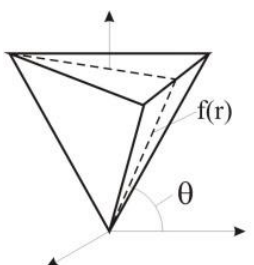

(a)

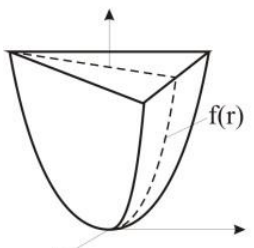

(d)

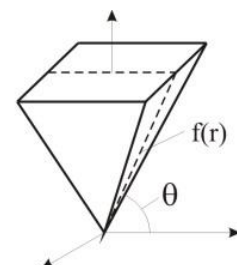

(b)

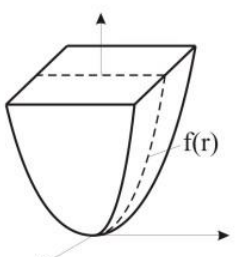

(e)

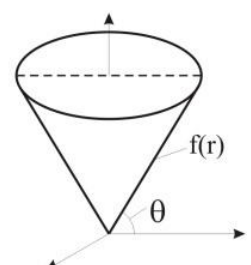

(c)

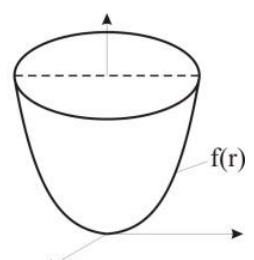

(f)

Fig. 3 Pyramid indenters for $n=1$ (a)-(c) and parabolic indenters for $n=2$ (d)-(f) with polygonal base, $m=3$ (triangle), $m=4$ (square), and $m=\infty$ (cycle) 
In [15], it was shown that an equivalent MDR-transformed profile does exist not only for axis-symmetrical indenters but also for indenters of arbitrary shape. As shown in [15] and [16], for this sake, quantity $l=k /\left(2 E^{*}\right.$ ) (where $k=\mathrm{d} F_{N} / \mathrm{d} d$ is the incremental normal stiffness) should be determined numerically as function of indentation depth $d$. Inverse function $d(l)$ is then exactly the unknown MDR transformed profile $g(x)$. Let us illustrate this simple procedure on the example of conical indenter. By differentiating Eq. (4) with respect of $d$ we get stiffness $k=4 E^{*} d /(\pi \tan \theta)$ and length $l=2 d /(\pi \tan \theta)$. Inverse relation $d=l(\pi / 2) \tan \theta$ coincides exactly with the MDR transformed profile (6). This procedure is applicable regardless of whether dependence $F_{N}(d)$ was obtained analytically, numerically or experimentally. In the following, we determine dependence $F_{N}(d)$ numerically and extract from it the MDR-transformed profiles for a number of tapered profiles with polygonal cross-sections (Fig. 3).

We start with consideration of pyramidal indenters. As shown in Fig. 3(a)(b), the bases of the indenter are regular polygons. Angle $\theta$ is defined as the angle between the ground plane and the 3D indenter side surface as shown in Fig.3.

In the simulation we calculated the contacts of pyramid indenters with different polygonal bases varying from $m=3$ to 20 , and for each type the angle ranges from $\theta=\pi / 64$ to $31 \pi / 64$. All the simulation results show that the one-dimensional profile is still a linear function which can be formulated as:

$$
g(x)=c_{1 \mathrm{D}} \cdot|x|
$$

with $c_{1 \mathrm{D}}$ :

$$
c_{1 \mathrm{D}}=\alpha \cdot \tan \theta
$$

where $\alpha$ is dependent only on polygon order $m$. For the sake of comparison we can define a fictive rotationally symmetric $3 \mathrm{D}$ profile with the same inclination angle:

$$
f_{3 \mathrm{D}}(r)=c_{3 \mathrm{D}} \cdot r=\tan \theta \cdot r .
$$

Then we can write $\alpha=c_{1 \mathrm{D}} / c_{3 \mathrm{D}}$. The values of $\alpha$ for different shapes of polygons are shown in Fig. 4(a) and Table 2. For a larger $m$, the shape of the pyramid indenter is close to a cone, the value of $\alpha$ is almost equal to $\pi / 2$.
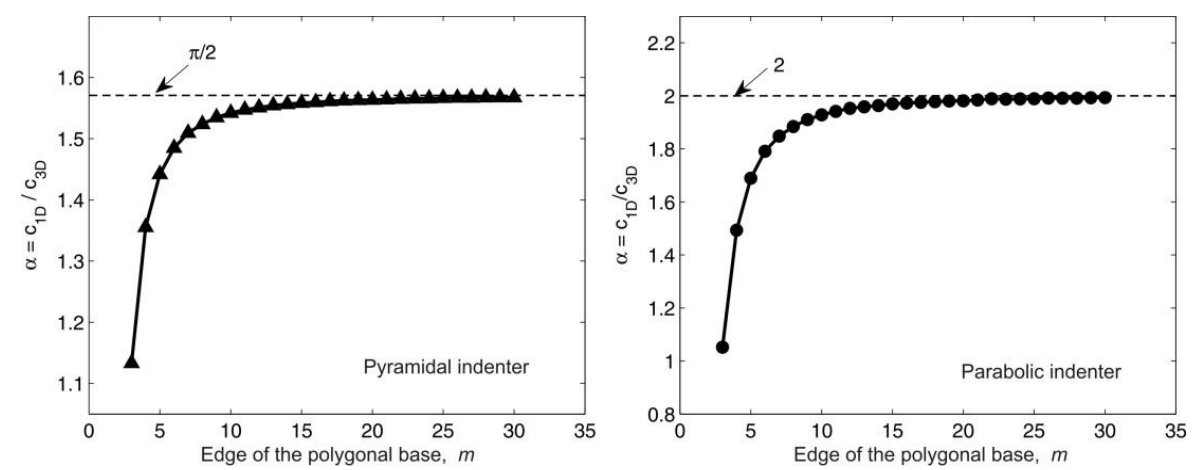

Fig. 4 Coefficient of $\alpha$ for pyramidal indenter $n=1$ (a) and parabolic indenter $n=2$ (b) with different polygonal bases 
Table 2 Values of coefficient $\alpha$

\begin{tabular}{cccccccccc}
\hline $\begin{array}{c}m \\
\text { polygon }\end{array}$ & $\begin{array}{c}3 \\
\text { triangle }\end{array}$ & $\begin{array}{c}4 \\
\text { square }\end{array}$ & 5 & 6 & 7 & 10 & 20 & 30 & $\begin{array}{c}\infty \\
\text { cycle }\end{array}$ \\
\hline $\begin{array}{c}\alpha(n=1) \\
\text { pyramid }\end{array}$ & 1.133 & 1.356 & 1.422 & 1.485 & 1.510 & 1.542 & 1.564 & 1.568 & $\pi / 2$ \\
$\begin{array}{c}\alpha(n=2) \\
\text { paraboloid }\end{array}$ & 1.052 & 1.493 & 1.690 & 1.791 & 1.848 & 1.928 & 1.981 & 1.993 & 2 \\
\hline
\end{tabular}

\subsection{Indenters with arbitrary power function geometry}

Let us now consider the case when the side surface of the indenter is not flat but is given by a power function. An example of parabolic indenter (shape with power 2 ) is shown in Fig. 3(d)-(f). We first remember the corresponding solution for an axisymmetric indenter with an arbitrary power function shape $f(r)=c_{n} \cdot r^{n}$. According to Eq. (5) its one-dimensional MDR-transformed profile is given by:

$$
g(x)=\kappa_{n} c_{n} \cdot|x|^{n},
$$

where:

$$
\kappa_{n}=\frac{\sqrt{\pi}}{2} \cdot \frac{n \Gamma(n / 2)}{\Gamma(n / 2+1 / 2)},
$$

and $\Gamma(n)$ is gamma function. In particular, for the cone $(n=1) \kappa_{1}=\pi / 2$ and for a paraboloid $(n=2) \kappa_{2}=2$, corresponding to $\alpha=c_{1 \mathrm{D}} / c_{3 \mathrm{D}}$ for $m=\infty$ as shown in Fig. 4 and Table 2.

As in the previous Section, we define an axis-symmetrical shape with the same power-law shape as shown in detail in Fig. 3. To underline that we have to do with a three-dimensional body which is in contact with a three-dimensional half-space, we denote the corresponding reference shape as

$$
f_{3 D}(r)=c_{3 D} \cdot r^{n} .
$$

This shape coincides with the vertical section of the polygonal indenters (shown by dashed lines in Fig. 3).

The numerical indentation tests were carried out for different indenters with power function $n$ from 1 to 20 and the polygonal base parameter $m$ from 3 to 30 . The results show that the 1D profile for an arbitrary power function is still a power function with the same power. Coefficient $\alpha=c_{1 \mathrm{D}} / c_{3 \mathrm{D}}$ for the same type of indenter (fixed $n$ and $m$ ) is constant (independent of coefficient $c_{3 \mathrm{D}}$ ). An example of parabolic indenter $(n=2)$ is shown in Fig. 4 (b), where the values of $\alpha$ for triangle, square and further polygonal based profile are presented. In the limiting case the indenter is a spherical cylinder, and $\alpha=2$ corresponding to $\kappa_{2}=2$ is well-known from the MDR theory [14].

If we use the following parameter instead of $\alpha$

$$
\xi=\frac{c_{1 D}}{\kappa_{n} c_{3 D}},
$$

then in the limiting case $m=\infty$, value $\xi$ for any power function $n$ will be equal to $1, \xi_{m=\infty}=1$. Some values of $\xi$, in particular for pyramid and parabolic indenter with triangle and square base are shown in Fig. 5 and Table 3. 


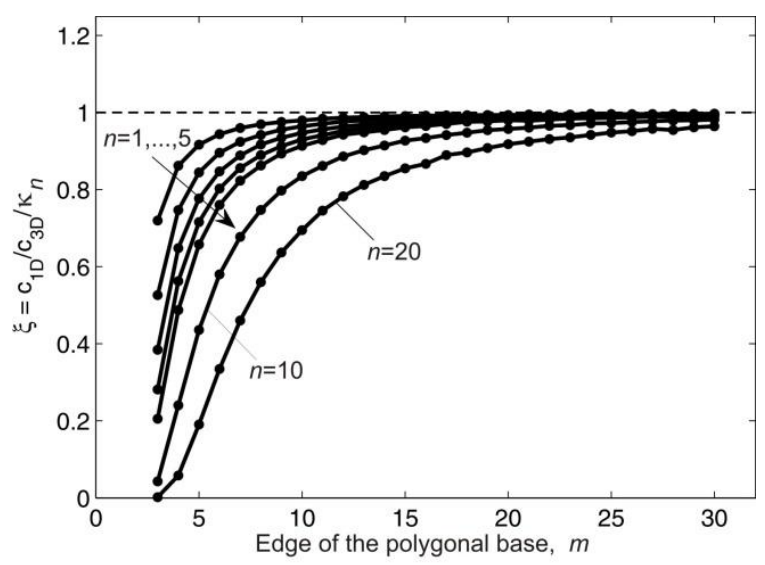

Fig. 5 Coefficient of $\xi$ for indenters with power function profile

Table 3 Values of coefficient $\xi$

\begin{tabular}{ccccccc}
\hline \multirow{2}{*}{$n$} & \multicolumn{7}{c}{$m$} \\
\cline { 2 - 7 } & $\begin{array}{c}3 \\
\text { (triangle) }\end{array}$ & $\begin{array}{c}4 \\
\text { (square) }\end{array}$ & 5 & 10 & 20 & 30 \\
\hline 1 (pyramid) & 0.723 & 0.866 & 0.923 & 0.986 & 1.000 & 1.000 \\
2 (paraboloid) & 0.526 & 0.747 & 0.845 & 0.964 & 0.991 & 0.997 \\
3 & 0.384 & 0.648 & 0.777 & 0.947 & 0.987 & 0.994 \\
10 & 0.043 & 0.241 & 0.058 & 0.835 & 0.957 & 0.983 \\
20 & 0.002 & 0.058 & 0.190 & 0.695 & 0.918 & 0.964 \\
\hline
\end{tabular}

\subsection{Consideration of indenters with the same base area}

In Section 2 it is found that the contact stiffnesses of triangular, rectangular indenters and flat cylinder with the same cross-section area are almost the same, and differ at most by $6 \%$. It thus appears to be sensible to try as "reference" indenters the axisymmetrical profiles with the same area of cross-section. This definition is slightly different from the definition in the previous Section. For both initial polygonal profile and the reference axisymmetrical profile we carry out the MDR transformation and determine the equivalent 1D-MDR profiles. Let us explain the exact procedure on the example of a pyramid indenter $(n=1)$. First, we determine the area of the indenter at different height and construct a cone with exactly the same cross-section areas. Then we carry out the three dimensional indentation test of the polygonal indenter by the BEM simulation and extract corresponding MDR profile $g(x)_{m \text {-poly }}$ and corresponding coefficient $c_{1 \mathrm{D}, m \text {-poly }}$ as described in Section 3 . For the reference axisymmetrical profile, the corresponding MDR transformed profile and the corresponding coefficient $c_{1 \mathrm{D}, m=\infty}$ are determined by (5). Finally we compare this $c_{1 \mathrm{D}, m \text {-poly }}$ and the coefficient of the axisymmetric conical profile using the ratio

$$
\zeta=\frac{c_{1 D, m \text {-poly }}}{c_{1 D, m=\infty}} .
$$


In an absolute similar way comparisons were also carried out for other power function geometries. The results are shown in Fig. 6 and Table 4. It can be seen that the coefficient $c_{1 \mathrm{D}}$ of pyramid indenter is close to that of conical indenter: it differs by at most $7 \%$ in the case of triangular base $\left(c_{1 \mathrm{D}}=0.927\right)$. It is noted that coefficient $c_{1 \mathrm{D}}$ cannot directly reflect the contact stiffness. Take an example of triangular indenter with power $n=20$ whose geometry is close to the flat triangular indenter (Fig.1a), its $\zeta$ is very small $\zeta=0.295(m=3, n=20)$, but the contact stiffness at the large indentation depth is the same to the flat indenter.

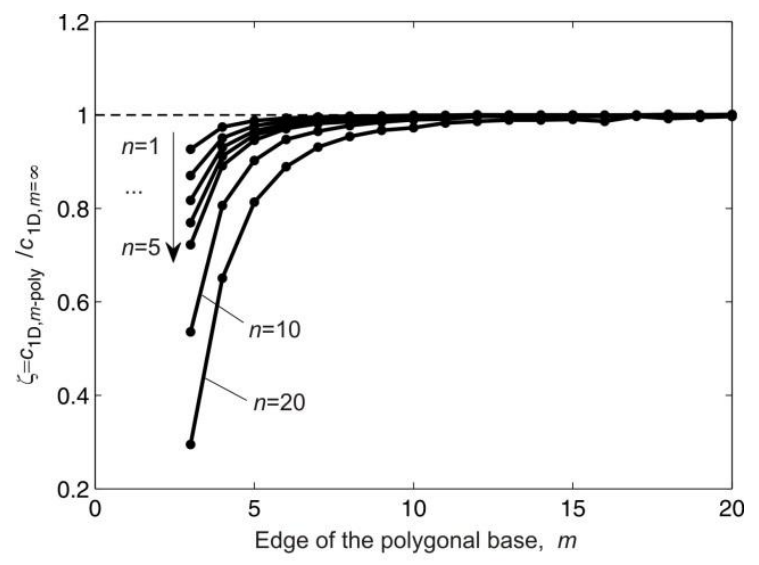

Fig. 6 Comparison of coefficient $c_{1 \mathrm{D}}$ among different indenters with the same base area

Table 4 Coefficient $\zeta$ for different power $n$ and polygon $m$

\begin{tabular}{ccccccc}
\hline \multirow{2}{*}{$n$} & \multicolumn{7}{c}{$m$} & & & \\
\cline { 2 - 7 } & $\begin{array}{c}3 \\
\text { (triangle) }\end{array}$ & $\begin{array}{c}4 \\
\text { (square) }\end{array}$ & 5 & 6 & 10 & 20 \\
\hline 1 (pyramid) & 0.927 & 0.974 & 0.988 & 0.994 & 1.000 & 1.000 \\
2 (paraboloid) & 0.870 & 0.951 & 0.977 & 0.988 & 0.997 & 1.000 \\
3 & 0.817 & 0.931 & 0.966 & 0.981 & 0.996 & 1.000 \\
10 & 0.536 & 0.806 & 0.820 & 0.893 & 0.990 & 1.000 \\
20 & 0.295 & 0.651 & 0.814 & 0.889 & 0.973 & 0.997 \\
\hline
\end{tabular}

\section{CONCLUSION}

Indentation of flat-ended and tapered indenters with polygonal base was numerically simulated using the boundary element method. The contact stiffnesses of prismatic punches with the same cross section area are almost same as the cylindrical indenter, where the triangular punch differs at most by $6 \%$. For pyramidal indenter and others with power function side, the one dimensional MDR transformed profile was generated based on the three dimensional simulation of indentation. It is found that the 1D profile is still a power function with the same power and it differs only by a constant factor. The factor was numerically calculated for the indenters with different power function side and different polygonal base. The generated MDR profiles can be used for the further contact problems, such as tangential contact or contact with linear viscoelastic bodies. 


\section{REFERENCES}

1. Oliver, W.C., Pharr, G.M., 2011, Measurement of hardness and elastic modulus by instrumented indentation: Advances in understanding and refinements to methodology, Journal of Materials Research, 19(1), pp. 3-20.

2. Fischer-Cripps, A.C., 2000, A review of analysis methods for sub-micron indentation testing, Vacuum, 58(4), pp.569-585.

3. Hay, J., Agee, P., Herbert, E., 2010, Continuous stiffness measurement during instrumented indentation testing, Experimental Techniques, 34, pp. 86-94.

4. Swadener, J.G., George, E.P., Pharr, G.M., 2002, The correlation of the indentation size effect measured with indenters of various shapes, Journal of the Mechanics and Physics of Solids, 50(4), pp. 681-694.

5. King, R.B., 1987, Elastic analysis of some punch problems for a layered medium, International Journal of Solids and Structures, 23(12), pp. 1657-1664.

6. Galin, L.A., 1961, Contact Problems in the Theory of Elasticity, North Carolina State College, USA

7. Sneddon, I.N., 1965, The relation between load and penetration in the axisymmetric Boussinesq problem for a punch of arbitrary profile, International Journal of Engineering Science, 23(12), pp. 1657-1664.

8. Oliver, W.C., Pharr, G.M., 1992, An Improved Technique for Determining Hardness and Elastic-Modulus Using Load and Displacement Sensing Indentation Experiments, Journal of Materials Research, 7(6), pp. 1564-1583.

9. Pharr, G.M., Oliver, W.C., Brotzen, F.R., 2011, On the generality of the relationship among contact stiffness, contact area, and elastic modulus during indentation, Journal of Materials Research, 7(3), pp. 613-617.

10. Popov V.L., 2010, Contact mechanics and friction: Physical principles and foundations, Springer, Berlin.

11. Pohrt, R., Li, Q., 2014, Complete boundary element formulation for normal and tangential contact problems, Physical Mesomechanics, 17(4), pp. 334-340

12. Pohrt, R., Popov, V.L., 2015, Adhesive contact simulation of elastic solids using local mesh-dependent detachment criterion in Boundary Elements Method, Facta Universitatis series: Mechanical Engineering, 13(1), pp. 3-10

13. Argatov, I., 2010, Frictionless and adhesive nanoindentation: Asymptotic modeling of size effects, Mechanics of Materials, 42(8), pp. 807-815.

14. Popov, V.L., Heß, M., 2015, Method of dimensionality reduction in contact mechanics and friction, Springer, Berlin.

15. Argatov, I., Heß, M., Pohrt, R., Popov, V.L., 2016, The extension of the method of dimensionality reduction to non-compact and non-axisymmetric contact, Journal of Applied Mathematics and Mechanics (ZAMM), 96(10), pp. 1144-1155.

16. Popov, V.L., Pohrt, R., Heß, M., 2016, General procedure for solution of contact problems under dynamic normal and tangential loading based on the known solution of normal contact problem, Journal of Strain Analysis for Engineering Design, 51(4), pp. 247-255. 